\title{
Biopsia hepática endoscópica transvaginal em cadelas
}

\author{
Endoscopic transvaginal liver biopsy in bitches
}

\author{
Leonardo Alves Coutinho Souza ${ }^{\mathrm{I}}$ Maurício Veloso Brun ${ }^{\mathrm{II}}$ Paula Cristina Basso ${ }^{\mathrm{III}}$ \\ Daniel Curvello de Mendonça Müller ${ }^{\mathrm{IV}}$ João Pedro Scussel Ferantiv Fabiane Reginatto dos Santosv \\ Marília Teresa de Oliveirav ${ }^{\mathrm{V}}$ Lucas Marques Colomév $^{\mathrm{L}}$ Luciana Hermes Dutra $^{\mathrm{II}}$
}

\section{RESUMO}

Esse trabalho teve o objetivo de propor uma técnica de biopsia hepática endoscópica transvaginal em cadelas, evitando-se o acesso através da parede abdominal, técnica essa internacionalmente conhecida por NOTES (natural orifice transluminal endoscopic surgery). Para tanto, foram utilizadas sete cadelas, as quais foram submetidas a dois procedimentos. O primeiro constou de biopsia hepática pela técnica proposta. Para isso, realizou-se a incisão vaginal após exteriorização através da vulva. Pela ferida vaginal foi introduzido um endoscópio flexível de $11 \mathrm{~mm}$ de diâmetro com dois canais de trabalho. Este foi empregado na obtenção das amostras hepáticas. $O$ segundo procedimento foi de avaliação laparoscópica aos 53 dias após a biopsia. As variáveis analisadas para a verificação de viabilidade, segurança $e$ qualidade dessa técnica foram: as dificuldades técnicas, as complicações cirúrgicas, o tempo operatório, a qualidade do material coletado, a formação de aderências vaginais $e$ as alterações bioquímicas hepáticas. Conclui-se que a biópsia hepática por NOTES flexível transvaginal é viável em cães, pois permite a obtenção de fragmentos apropriados para o exame histológico sem ocasionar alterações significativas dos parâmetros de avaliação hepática e formação de aderências intraperitoneais.

Palavras-chave: cão, videocirurgia, endoscopia, laparoscopia, NOTES.

\begin{abstract}
A technique of hepatic biopsy by transvaginal approach (NOTES - Natural Orifice Transluminal Endoscopic Surgery) is proposed. Seven dogs were used, and the experiment was divided into two stages including the liver biopsy and the evaluation of laparoscopic abdominal conditions after biopsy (53 days after). The animals were submitted to vaginal incision after exposition through the vulva. Through the vaginal incision was introduced a flexible endoscope of $11 \mathrm{~mm}$ with two working channels, was introduced through the vaginal incision and used to obtain liver samples. The variables analyzed to verify the feasibility of the proposed biopsy were: operative time, surgical complications, technical difficulties, the quality of sample collected, the formation of vaginal adhesions and their characteristics and the biochemical condition of the liver. In conclusion, the liver biopsy by endoscopic transvaginal access is feasible in dogs. It permits to take appropriated hepatic samples for histological studies without changinghepatic parameters and intraperitoneal adhesions.
\end{abstract}

Key words: dog, videosurgery, endoscopy, laparoscopy, liver biopsy, NOTES.

\section{INTRODUÇÃO}

A cirurgia endoscópica transluminal por orifícios naturais (NOTES) pode combinar duas

\footnotetext{
IPrograma de Pós-graduação em Cirurgia e Anestesiologia Veterinária, Universidade de Franca (UNIFRAN), Franca, MG, Brasil. IIDepartamento de Clínica de Pequenos Animais, Centro de Ciências Rurais (CCR), Universidade Federal de Santa Maria (UFSM), Santa Maria, RS, Brasil.

IIIPrograma de Pós-graduação em Medicina Veterinária (PPGMV), CCR, UFSM, Santa Maria, RS, Brasil.

${ }^{\text {IV }}$ Departamento de Estudos Agrários (DEAG), Universidade Regional do Noroeste do Estado do Rio Grande do Sul (UNIJUÍ), Ijuí, RS, Brasil. Endereço para correspondência: Rua Reinaldo Manoel Guidolin, 257/302, 97110-212. E-mail: cmdaniel@terra.com.br. vDepartamento de Clínica de Pequenos Animais, Faculdade de Medicina Veterinária e Agronomia (FAMV), Universidade de Passo Fundo (UPF), Passo Fundo, RS, Brasil.
} 
abordagens cirúrgicas convencionais guiadas por imagem: a endoscopia flexível e a cirurgia laparoscópica, culminando na modalidade cirúrgica com uma característica principal: ausência de incisões abdominais (BARON, 2007). Vários procedimentos cirúrgicos de diferentes graus de complexidade já foram realizados com essa técnica, como apendicectomia, colecistectomia, ovariectomia, ovário-histerectomia (OSH) e colheita de amostras (KALLO et al., 2004).

A primeira descrição de NOTES em animais foi realizada por KALLO et al. (2004) que obtiveram o acesso à cavidade peritoneal por via transgástrica em suínos, com posterior colheita de biopsia hepática e fechamento da cavidade gástrica com grampos cirúrgicos. Já em humanos, a primeira colecistectomia transvaginal, sem auxílio de laparoscopia, foi descrita por ZORRON et al. (2008).

WAGG et al. (2006) e MCGEE et al. (2006) descreveram como vantagens potenciais na aplicação da NOTES o fato de operar com menores graus de invasão e traumatismo abdominal, assim como a queda significativa de infecção hospitalar e do tempo de convalescença. KALLO et al. (2004) apontaram como ponto positivo, a ausência de cicatriz cirúrgica, enquanto KANTSEVOY et al. (2005) ressaltaram como vantagens principais da técnica o baixo risco de infecções e de herniações pós-operatórias.

A biópsia hepática consiste no principal procedimento para a obtenção do diagnóstico definitivo e o prognóstico da maioria das hepatopatias. A escolha da técnica para coleta tecidual depende de uma série de fatores, tais como a provável doença hepática, a condição geral do paciente, a disponibilidade de equipamentos apropriados e equipe técnica treinada (BRAVO et al., 2001). Apesar de existirem diferentes possibilidades técnicas para a execução de biópsia hepática em cães, se faz necessário expandir o campo de investigação no que se refere à busca de novos procedimentos ainda menos invasivos. Considerando a importância que a NOTES vem demonstrando na cirurgia experimental, aliada à constante necessidade de obtenção de biopsias hepáticas em caninos, objetivou-se testar essa nova modalidade cirúrgica como possível via para coleta de tecido hepático em cadelas pelo acesso transvaginal.

\section{MATERIAL E MÉTODOS}

Foram utilizadas sete cadelas adultas, pesando de 6 a 9kg, não castradas, sem raça definida, provenientes de uma Associação de Proteção dos Animais. As cadelas foram avaliadas clinicamente, desverminadas e alojadas em canis individuais.
Anteriormente ao procedimento cirúrgico, todos os animais foram submetidos a jejum alimentar sólido de 12 horas e hídrico de quatro horas. Realizou-se a tricotomia da região abdominal, que se estendeu do apêndice xifóide ao púbis.

Para o procedimento cirúrgico, os animais foram pré-medicados com diazepam (0,5 $\mathrm{mg} \mathrm{kg}^{-1}$, IM) e submetidos à anestesia epidural com a associação de sulfato de morfina (0,1 $\left.\mathrm{mg} \mathrm{kg}^{-1}\right)$ e lidocaína (4mg kg-1). Na seqüência, foram induzidos à anestesia geral, por meio da vaporização de isofluorano em máscara facial, em máxima concentração. Durante todo o procedimento manteve-se a canulação venosa com infusão de ringer lactato, na velocidade de $10 \mathrm{ml} \mathrm{kg}^{-1} \mathrm{~h}^{-1}$. A manutenção da anestesia foi obtida com isofluorano vaporizado em oxigênio a 100\%, em circuito circular semi-fechado, com respiração assistida. Como quimioprofilaxia antimicrobiana, utilizou-se ampicilina sódica (20 $\mathrm{mg} \mathrm{kg}^{-1}$, IV) e enrofloxacina ( $\left.5 \mathrm{mg} \mathrm{kg}^{-1}, \mathrm{IV}\right)$, administradas 30 minutos antes do procedimento cirúrgico.

Com os pacientes posicionados em decúbito dorsal, promoveu-se a anti-sepsia da parede abdominal com solução de iodo polivinilpirrolidona (PVP-I) degermante, seguido de PVP-I alcoólico a 10\%. A cavidade vaginal e a vulva foram preparadas com solução de PVP-I aquoso a 0,1\% $\left(10 \mathrm{ml} \mathrm{kg}^{-1}\right)$. Para o acesso vaginal, todos os animais foram submetidos previamente à sondagem uretral promovendo-se total esvaziamento da bexiga. O abdômen foi então insuflado com $\mathrm{CO}_{2}$, a partir da punção abdominal com agulha de Veress, na velocidade de $2 \mathrm{~L} \min ^{-1}$ até se alcançar a pressão de $12 \mathrm{mmHg}$. Com o uso de um espéculo vaginal foi apreendido o fundo da vagina junto à região da cérvix com pinças hemostáticas Kelly retas e longas. Com tesoura de Metzembaum convencional realizouse incisão da mucosa vaginal, seguida da dissecação da musculatura da vagina até se obter o acesso à cavidade pélvica.

Na seqüência, um endoscópio flexível de $11 \mathrm{~mm}$ de diâmetro foi então posicionado através da ferida vaginal. Quando necessário, ampliou-se a velocidade de insuflação pela agulha, para contrapor o escape de gás pela ferida vaginal. O endoscópio foi então direcionado para região hepática, elegendo-se o ponto de biopsia em dois diferentes lobos hepáticos. Para a coleta do fragmento hepático (Figura 1) foi utilizada uma pinça endoscópica tipo hot-biopsy. O material foi devidamente colhido, identificado e fixado em formol. Por fim, o endoscópio foi removido e procedeu-se a desinsuflação da cavidade abdominal. O fundo da vagina não foi suturado, optando-se pela cicatrização por segunda intenção. No período pósoperatório os animais foram medicados com 


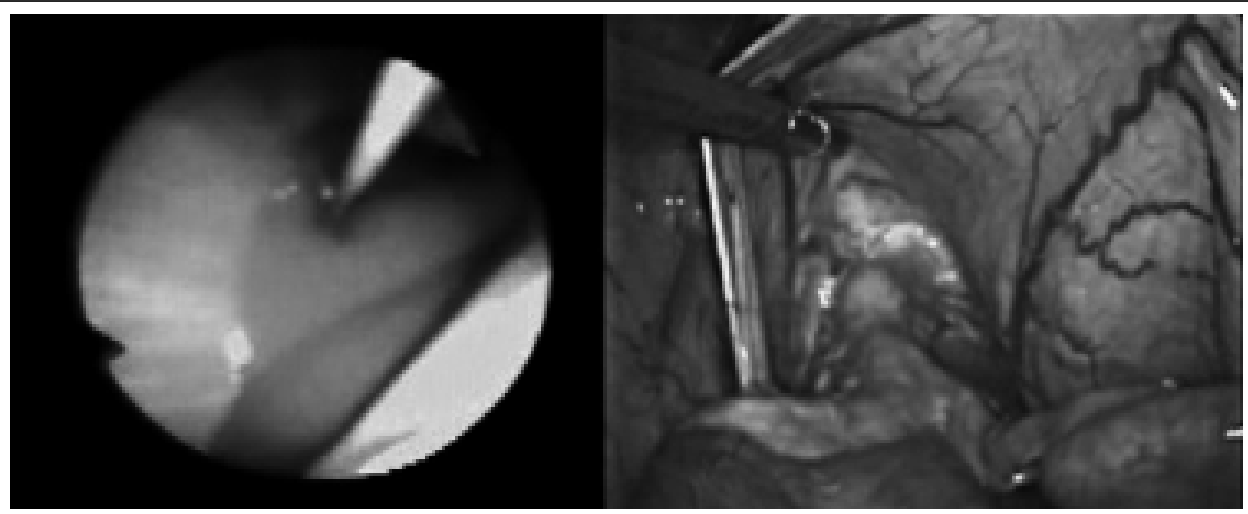

Figura 1 - Biópsia hepática por Notes transvaginal no animal 2 (imagem da esquerda). Visualização da região de acesso vaginal no animal 7 submetido à biópsia hepática por NOTES vaginal com endoscópio flexível. Verificou-se a inexistência de quaisquer aderências pós-cirúrgicas entre a vagina e a bexiga ou entre a vagina e o reto (imagem da direita).

enrofloxacina (5mg kg-1, VO, SID) por sete dias, cloridrato de tramadol (2mg kg-1, SC, TID) e carprofeno $\left(4,4 \mathrm{mg} \mathrm{kg}^{-1}\right.$, VO, SID) durante três dias. As biopsias foram incluídas em parafina, coradas pela hematoxilina eosina, e avaliadas por microscopia óptica.

Com o intuito de avaliar a incisão vaginal, a formação de aderências abdominais e as possíveis alterações relacionadas à biopsia hepática, foram realizadas laparoscopias exploratórias aos 53 dias após o procedimento, precedendo a realização de $\mathrm{OSH}$ laparoscópica para castração. Para tanto, as cadelas foram submetidas ao mesmo protocolo anestésico descrito anteriormente, realizando-se a laparoscopia seguida da técnica de OSH com três portais, descrita por BRUN et al. (2004). A cavidade foi cuidadosamente inspecionada, registrando-se eventuais alterações nas diferentes estruturas anatômicas e se avaliando a condição da região na qual se produziu a ferida vaginal de acesso. Assim, buscava-se identificar as características das aderências existentes, empregandose o escore previamente descrito por SILVA et al. (2007).

A aplicabilidade da NOTES foi avaliada pelo tempo cirúrgico, pela possibilidade de observação hepática, coleta de material apropriado para biópsia hepática, ocorrência de complicações ou intercorrências, dificuldades técnicas e formação de aderências vaginais e/ou abdominais. Também foi realizada a avaliação sérica da função hepática, pela dosagem de alanina aminotransferase (ALT), fosfatase alcalina (FA), gama glutamiltransferase (GGT) e albumina, nos seguintes tempos: T1 (antes do procedimento de biópsia - valores basais); T2 (ao término da operação), T3 (48h após a operação), T4 (oito dias após a operação) e T5 (53 dias após a operação). Para essas variáveis aplicou-se o teste de Wilcoxon. Os resultados foram considerados significativos quanto $\mathrm{P} \leq 0,05$.

\section{RESULTADOS E DISCUSSÃO}

O tempo médio da realização do procedimento cirúrgico, desde a incisão da mucosa vaginal até a completa drenagem do pneumoperitônio

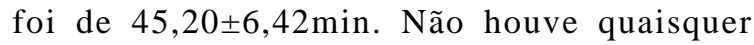
intercorrências anestésicas e o protocolo empregado demonstrou ser eficaz para a execução do procedimento proposto.

Optou-se pela realização do pneumoperitônio previamente ao acesso vaginal na busca de se promover afastamento das vísceras abdominais em relação à parede abdominal previamente à introdução do endoscópio. Para tanto, utilizou-se a agulha de Veress, a qual ficou posicionada no abdome durante toda a operação para manter a insuflação peritoneal na pressão pré-estabelecida. Foram verificadas duas intercorrências associadas ao emprego da agulha de Veress. Em um dos animais, verificou-se a punção seromuscular do cólon descendente durante o início do pneumoperitônio. Em outro deles, houve punção esplênica, a qual não ocorreu durante o estabelecimento do pneumoperitônio, e sim pela movimentação da agulha. Em ambos os casos houve resolução espontânea e não estiveram associados a alterações clínicas ou hematológicas no período pós-operatório. Verificouse na laparoscopia, aos 53 dias após a biopsia, que o mesométrio aderiu-se ao cólon desse animal. Acreditase que isso tenha ocorrido junto ao local de punção, e 
que esse tecido tenha se comportado como o omento, o qual tende a se aderir naturalmente em áreas lesionadas ou desnudas de peritônio (HENDERSON, 1982).

Considerando que a insuflação do abdome, previamente à introdução do endoscópio flexível, não propiciou maior segurança quanto à prevenção de lesões orgânicas de acesso, os autores acreditam que a insuflação possa ser estabelecida com melhores resultados através do endoscópio, após vencida a etapa de acesso vaginal. Para tanto, uma possibilidade seria empregar o próprio canal de insuflação do endoscópio, condição essa que não foi testada na técnica proposta.

$\mathrm{O}$ acesso à cavidade abdominal por via transvaginal foi considerado de média complexidade pela equipe cirúrgica, não havendo intercorrência na maior parte dos pacientes (cinco animais). Contudo, não foi possível definir exatamente o posicionamento de entrada na cavidade pélvica, pois a partir da dissecação da parede vaginal produzia-se um trajeto muscular/submucoso comunicando a incisão da mucosa com a do peritônio, sem que fosse possível controlar adequadamente a sua extensão. Isso ocorreu devido à flexibilidade da parede vaginal e a dificuldade de definição dos planos de dissecação, além da hemorragia que se apresentava de baixo volume, porém constante.

Em três animais houve lesões de acesso associadas à produção da ferida na serosa da vagina. Em duas, dessas três cadelas, verificou-se pequena hemorragia da parede muscular da vagina ao se promover o acesso dorsolateral em relação à cervix do lado esquerdo, sendo necessário alterar a posição para o lado direito e manter compressão temporária com gaze cirúrgica para controlar o sangramento. Na outra fêmea, ocorreu lesão seromuscular do reto na etapa de confecção da ferida serosa, a qual também foi realizada do lado esquerdo. Ao se verificar que o intestino estava lesionado, realizou-se uma sutura seromuscular com um único ponto isolado simples, utilizando-se fio de poliglactina 910 n.3-0. Na sequência, alterou-se o local de acesso vaginal, realizando-se a ferida do lado direito, seguida da dissecação parcial da parede da vagina, o suficiente para permitir que o endoscópio fosse alojado através da ferida. O endoscópio foi então introduzido através da vagina de forma contusa, sendo empurrado para o interior da cavidade, concluindo a dissecção da parede vaginal e promovendo ruptura da serosa. Ao final da biopsia, utilizou-se o próprio endoscópio para a realização de uma colonoscopia, condição que permitiu a visualização da integridade da mucosa retal no local onde houve a ferida seromuscular, sendo que a sutura utilizada na reconstrução não alcançava a mucosa retal. A partir desse acidente, procurou-se posicionar o endoscópio nos demais animais de forma romba, sem a realização de incisão através da serosa, posicionando-o craniolateralmente do lado direito em relação à cervix das cadelas, já que o cólon descendente anatomicamente está localizado a esquerda do paciente (EVANS \& LAHUNTA, 1994), evitando maior risco de lesão intestinal.

O fato da lesão retal no acesso vaginal ter ocorrido no terceiro paciente operado e não ter sido observada nas demais cadelas, ressalta quanto à importância de transpassar a curva de aprendizado durante a execução da NOTES, para se reduzir os riscos de complicações cirúrgicas, tal como é amplamente descrito por SCHWANDNER et al. (1999) em relação à cirurgia laparoscópica. O que evitou a contaminação com material fecal foi a projeção da mucosa colônica observada através da ferida seromuscular do órgão, condição que naturalmente ocorre em cirurgias intestinais, quando existe secção, pelo menos parcial, da submucosa (FOSSUM et al., 2002).

No momento da introdução romba do endoscópio, verificou-se que dois animais contraíram o membro posterior direito, associando-se essa condição com possível compressão de nervo periférico. No pós-operatório dessas pacientes não se verificou qualquer grau de claudicação ou outro comprometimento sistêmico associado a essa observação. Dessa maneira, os autores passaram a realizar a introdução do endoscópio de forma romba a partir da ferida vaginal, contudo, direcionando-o crânio-lateralmente em angulação aproximada de $45^{\circ}$ em relação à linha média ventral, desviando-se da bexiga e do cólon descendente, e evitando assim o contato com a parede muscular e gordura pélvica com suas terminações nervosas associadas.

Em todos os animais foi possível a visualização ampla da superfície peritoneal e nítida inspeção hepática. O acesso possibilitou a avaliação da bexiga, parte dos cornos uterinos, das alças intestinais, do fígado, da vesícula biliar, do baço, do estômago, dos rins e da superfície diafragmática. Em todas as cadelas obtiveram-se, sem maiores dificuldades técnicas, duas amostras hepáticas de diferentes lobos. No entanto, a ausência de instrumental auxiliar para manipulação de órgãos não permitiu maior exposição desses sob o epíplon (considerando o paciente em decúbito dorsal), incluindo parte dos cornos uterinos e ovários. Além disso, a exposição dos lobos hepáticos laterais direito e esquerdo foi mais bem obtida lateralizando-se os pacientes, a partir do posicionamento do membro anterior correspondente no sentido contrário ao lobo a ser inspecionado. 
Nenhum animal apresentou náusea/vômito e diarréia no período pós-operatório. Uma cadela (18\%) apresentou no sétimo dia de pós-operatório secreção vaginal serosa e sinais de dor à micção (05). Pela urinálise diagnosticou-se cistite e a paciente foi tratada com antibioticoterapia sistêmica a base de enrofloxacina ( $5 \mathrm{mg} \mathrm{kg}^{-1}$, BID), por sete dias, além de analgésicos a base de cloridrato de tramadol (2mg kg-1, TID, por três dias), apresentado completa resolução do quadro clínico após o tratamento. Acredita-se que a cistite possa ter sido originada de uma vaginite bacteriana pós-operatória, ou até mesmo por retenção urinária (WARE, 2006). Suspeita-se que a extensão das lesões vaginais nessa cadela possa ter sido associada a desconforto miccional no pós-operatório, o que por sua vez poderia ocasionar a retenção urinária.

Em relação aos valores médios de ALT mensurados, esses se mantiveram dentro dos parâmetros normais para a espécie durante todos os tempos avaliados (FELDMAN et al., 2000). Contudo, houve uma diminuição significativa de T1 (basal) para o T2 (término da operação) $(P=0,018)$. Ocorreu um aumento significativo da ALT em T3 (48hs após a biópsia) em relação à T2 $(P=0,037)$. Em T4 (oito dias após biópsia), houve diminuição significativa desse parâmetro em relação a T3( $P=0,029)$. Já em T5 (53 dias após biópsia), não ocorreram alterações significativas em relação a T4 $(P=0,249)$ (há que se comparar T2, T3, T4 e T5 com T1, que é o basal). Sabendo-se que a ALT é um indicativo de lesão hepática (LOPES et al., 2007), possivelmente se pode atribuir essa pequena elevação da enzima pelo próprio procedimento de biópsia, o qual ocasionou reação inflamatória, ainda que mínima, junto ao sítio de coleta. Esse fato pode ser evidenciado nas primeiras 48 horas pós-operatórias (T3), mas que regrediu oito dias após a operação (T4).

Os valores da FA estiveram dentro dos parâmetros normais para a espécie durante todos os tempos avaliados (FELDMAN et al., 2000). Porém, em relação aos valores basais, (T1) verificou-se elevação significativa desse parâmetro em T3 (48hs após operação) e T4 (oito dias após) ( $P=0,029$, e $P=0,037$, respectivamente). Já de T4 para T5, verificou-se que a FA diminuiu significativamente $(P=0,037)$. A elevação significativa da FA em T3 em relação aos valores basais (T1) demonstra que possa ter havido leve colestase, possivelmente decorrente do processo inflamatório (LOPES et al., 2007) ocasionado pela biópsia hepática.

Todos os valores médios de GGT estiveram dentro dos limites considerados normais para cães (FELDMAN et al., 2000). Em T2 houve aumento significativo em relação a T1 $(P=0,036)$, existindo aumento significativo entre $\mathrm{T} 4$ e os valores basais
$(P=0,044)$. Por fim, considera-se que a elevação dos valores médios da GGT a partir de T2 possa estar associada à mesma causa que promoveu a elevação da FA, conforme descreveram LOPES et al. (2007).

Assim como o constatado nas demais variáveis associadas às condições hepáticas, os valores médios de albumina mantiveram-se dentro dos limites fisiológicos segundo FELDMAN et al. (2000). Em T3 ocorreu diminuição significativa quando comparado aos valores basais $(P=0,017)$. Contudo, ao confrontar T3 com T4 e T5, verificou-se elevação significativa dessa variável $(P=0,011)$. Em relação à albumina, verificou-se redução significativa dessa proteína em relação aos níveis basais também em T3, alcançando-se valor médio no limite inferior considerado normal para a espécie (LOPES et al., 2007). É possível que isso tenha ocorrido devido à lesão hepática ocasionada pelo procedimento cirúrgico, pela possível esteatose leve verificada a partir das avaliações da FA e GGT, e pela própria condição do fígado dos pacientes experimentais, já que dos cinco animais que apresentaram redução da albumina em T3, dois demonstraram algum grau de esteatose e um possuía degeneração hidrópica, segundo os resultados das biopsias. Em T4, os valores retornaram aos níveis basais, demonstrando completa resolução da condição.

Considerando que os valores das avaliações bioquímicas se encontraram dentro dos limites considerados normais da espécie, e que nenhuma das alterações em relação aos tempos avaliados apresentou qualquer significado clínico, os autores consideram que a técnica proposta de biopsia por NOTES vaginal flexível, com o auxílio de pinça hotbiopsy, não ocasiona alterações hepáticas importantes, podendo ser utilizada para a coleta de fragmentos hepáticos.

A grande quantidade de amostra de biopsia associada com esmagamentos (cinco animais- 71\%) ocasionou intensa formação de artefatos. Acredita-se que os esmagamentos das amostras estejam diretamente associados ao instrumental utilizado. Deve ser considerado que a pinça empregada é, em sua origem, projetada para coleta de mucosa e submucosa do trato digestivo de pacientes humanos e animais (TAMNS, 1999), e não de tecido hepático, o qual possui consistência completamente diferenciada (JUNQUEIRA \& CARNEIRO, 2004).

Coloca-se que, mesmo que em todos os casos tenha sido possível avaliar adequadamente os hepatócitos, pelo menos em um animal (primeiro paciente) o material poderia ter sido insuficiente, pois foi constituído de único lóbulo hepático incompleto. Indica-se então, que seja coletado maior número de 
amostras hepáticas ao utilizar a técnica descrita, pois em condições nas quais existem áreas previamente lesionadas (como focos tumorais, esteatoses, lipidoses ou focos necróticos), nas quais o tecido hepático é mais frágil quando comparado aos de pacientes hígidos (MACLACHLAN \& CULLEN, 1998), tanto a quantidade como o esmagamento da amostra poderiam comprometer o resultado diagnóstico final.

A NOTES flexível pelo acesso vaginal permitiu, na grande maioria dos pacientes, a coleta de fragmentos de tamanho suficiente para a obtenção de diagnóstico histológico. Em apenas um animal não foi possível obter um lóbulo hepático completo, contudo, a amostra foi suficientemente grande para se constatar a presença de esteatose hepática. Nos outros seis animais (86\%) foram coletados dois ou três lóbulos hepáticos completos. Em cinco pacientes (71\%) houve maceração de leve a intensa das bordas da amostra, observando-se intensos artefatos associados ao esmagamento e a maceração.

A videolaparoscopia se mostrou excelente ferramenta para a avaliação das alterações pósoperatórias associadas a NOTES. Apesar da pequena variedade e quantidade de aderências observadas na laparoscopia, não houve manifestação de sinais clínicos sugestivos de enfermidades secundárias. Dentre as sete cadelas, quatro não apresentaram aderências (Figura 1). Um animal (14\%) apresentou aderência de omento com a superfície ventral da bexiga e um animal (14\%) apresentou adesão do ligamento falciforme. Um animal (14\%) possuía aderências de omento na linha média ventral, e extensas áreas de fibrose na parede lateral direita. Possuía também sinais de peritonite verificados pela presença de áreas de fibrose superficial junto à superfície peritoneal, principalmente na parede abdominal lateral direita. Em um dos animais verificou-se a presença de piometra durante a avaliação laparoscópica. Foi então submetido ao procedimento de OSH videoassistida, tendo alta em sete dias.

\section{CONCLUSÃO}

A técnica de biopsia hepática por NOTES vaginal com endoscópio flexível é viável em cadelas, pois permite a obtenção de fragmentos apropriados para o exame histológico, não ocasiona alterações significativas dos parâmetros de avaliação hepática e resulta em mínima formação de aderências intraperitoneais.

A maior dificuldade técnica é o acesso vaginal, o qual necessita melhorias a fim de minimizar os riscos trans-operatórios, indicando-se assim, a realização do acesso mais profundo possível na vagina, buscando-se a introdução do endoscópio cranialmente a gordura pélvica.

\section{COMITÊ DE ÉTICA E BIOSSEGURANÇA}

O experimento foi aprovado pelo Comitê de Ética Animal da Universidade de Franca sob o registro 001/2010.

\section{REFERÊNCIAS}

BARON, T.H. Natural orifice transluminal endoscopic surgery. Baron Joe Surg, v.94, n.1, p.1-2, 2007. Disponível em: $<$ http://onlinelibrary.wiley.com/doi/10.1002/bjs.5681/ abstract; jsessionid=FD 724D9A25DEB 9EB 88750E $01 \mathrm{~B} 51172 \mathrm{FC}$.d $04 \mathrm{t} 04$ ? system Message $=W$ il ey + Online+Library+will+be+disrupted $+5+$ Nov +from $+10-$ $12+\mathrm{GMT}+$ for+monthly+maintenance $>$. Acesso em: 03 nov. 2011. doi:10.1002/bjs.5681.

BRAVO, A.A. et al. Liver biopsy. New England Journal of Medicine, v.344, n.7, p.495-500, 2001.

BRUN, M.V. et al. Ovário-histerectomia laparoscópica com três portais em cães. In: CONGRESSO DO COLÉGIO BRASILEIRO DE CIRURGIA E ANESTESIOLOGIA VETERINÁRIA, 4., 2004, Indaiatuba. Brazilian Journal of Veterinary Research and Animal Science, v.41. p.153$154,2004$.

EVANS, H.E.; LAHUNTA. A. Miller: guia para dissecção do cão. 5.ed. Rio de Janeiro: Guanabara-Koogan, 2001. 250p.

FELDMAN, B.F. et al. Schalm's veterinary hematology. 5.ed. Philadelphia: Lippincott Williams \& Wilinks, 2000. 1344p.

FOSSUM, T.H. et al. Cirurgia do sistema digestório. In: Cirurgia de pequenos animais. São Paulo: Roca, 2002. Cap.16, p.222-405.

HENDERSON, R.A. Controlling peritoneal adhesions. Veterinary Surgery, v.11, n.1, p.30-36, 1982.

KANTSEVOY, S.V. et al. Endoscopic gastrojejunostomy with survival in a porcine model. Gastrointestinal Endoscopy, v.60, n.2, p.287-292, 2005. Disponível em: <http:// www.giejournal.org/article/S0016-5107(05)01565-8/abstract>. Acesso em: 03 nov. 2011.

LOPES, S.T.A. et al. Manual de patologia clínica veterinária. Santa Maria: UFSM/Departamento de Clínica de Pequenos Animais, 2007. 107p.

MACLACHLAN, N.J.; CULLEN, J.M. Fígado, sistema biliar e pâncreas exócrino. In: CARLTON. W.W; MCGAVIN, M.D. Patologia veterinária especial. 2.ed. São Paulo: Manole, 1998. p.95-131.

MCGEE, M.F. et al. A primer on natural orifice transluminal endoscopic surgery: building a new paradigm. Surgical Innovation, v.13, n.2, p.86-93, 2006. Disponível em: <http:/ /sri.sagepub.com/content/13/2/86.long>. Acesso em: 03 nov. 2011. doi: $10.1177 / 1553350606290529$. 
SCHWANDNER, O. et al. Advanced age-indication or contraindication for laparoscopic colorectal surgery. Diseases of the Colon \& Rectum, v.42, n.3, p.356-362, 1999. Disponível em: <http://www.ncbi.nlm.nih.gov/pubmed/ 10223756>. Acesso em: 03 nov. 2011.

SILVA, M.A.M. et al. Aderências intraperitoneais em candelas ovariohisterectomizadas: influência do omento na prevenção. In: CONFERÊNCIA LATINO-AMERICANA DE MEDICINA VETERINÁRIA, 2007, Rio de Janeiro. Anais... Rio de Janeiro: Revista Universidade Rural, v.27, suppl., p.317-319, 2007.

TAMNS, T.R. Endoscopic removal of gastrointestinal foreingn bodies. In: TAMNS, T.R; RAWLINGS, C.A. Small animal endoscopy. 2.ed. St. Louis: Elsevier - Health Sciences Division, 1999. Cap.8, p.247-298.
WAGH, M.S. et al. Survival studies after endoscopic transgastric oophorectomy and tubectomy in porcine model. Gastrointestinal Endoscopy, v.63, n.3, p.473-478, 2006. Disponível em: <http://www.giejournal.org/article/S00165107(05)02316-3/abstract>. Acesso em: 03 nov. 2011. doi:10.1016/j.gie.2005.06.045.

WARE, W.A. Distúrbios do trato urinário. In: NELSON, R.W.; COUTO, C.G. Medicina interna de pequenos animais. 3.ed. Rio de Janeiro: Elsevir, 2006. Cap.13, p.547- 561.

ZORRÒN, R. et al. NOTES transvaginal cholecystectomy: preliminary clinical application. Surgical Endoscopy, v.22, n.2, p.542-547, 2008. Disponível em: <http://www.springerlink.com/ content/pk3r8812q35126tx/>. Acesso em: 03 nov. 2011. doi: 10.1007/s00464-007-9646-5. 